\title{
Performance Prediction of Concrete Elements in Bridge Substructures using Integrated Deterioration Method
}

\author{
Guoping BU \\ PhD Candidate, \\ Griffith University, \\ QLD, Australia \\ g.bu@griffith.edu.au
}

Michael BLUMENSTEIN

Associate Professor

Griffith University,

QLD, Australia

m.blumenstein@griffith.edu.au

\author{
Jaeho LEE \\ Research Fellow \\ Griffith University, \\ QLD, Australia \\ j.lee@griffith.edu.au
}

\author{
Yew-Chaye LOO \\ Professor \\ Griffith University, \\ QLD, Australia \\ y.loo@griffith.edu.au
}

\author{
Hong GUAN \\ Associate Professor \\ Griffith University, \\ QLD, Australia \\ h.guan@griffith.edu.au
}

\section{Summary}

The typical probabilistic deterioration model cannot guarantee a reliable long-term prediction for various situations of available condition data. To minimise this limitation, this paper presents an advanced integrated method using state-/time-based model to build a reliable transition probability for prediction long-term performance of bridge elements. A selection process is developed in this method to automatically select a suitable prediction approach for a given situations of condition data. Furthermore, a Backward Prediction Model (BPM) is employed to effectively prediction the bridge performance when the inspection data are insufficient. In this study, a benchmark exampleconcrete element in bridge substructures is selected to demonstrate that the BPM in conjunction with time-based model can improve the reliability of long-term prediction.

Keywords: probabilistic deterioration model; integrated method; transition probability; long-term performance; Backward Prediction Model (BPM); time-based model; state-based model.

\section{Introduction}

Reliable decision making of a bridge Maintenance, Repair and Rehabilitation (MR\&R) activities is highly dependent on the reliable current year's inspection data and well-estimated future structure conditions. To achieve cost-effectiveness of MR\&R during bridge service life, developing a reliable deterioration model is essential. A large number of historical bridge condition ratings are usually required for a reliable deterioration model in a bridge management system (BMS) to attain best possible outcomes. However, some bridge agencies may not have sufficient historical bridge inspection records to achieve the basic requirement of running a deterioration model in the current BMSs. Thus, development of an advanced bridge deterioration model to predict reliable long-term bridge performance is an issue of utmost urgency.

The difficulty in developing a reliable deterioration model is that bridge condition is mostly inspected using a quantitative method on a discrete scale, which implies inherent uncertainty in bridge deterioration conditions. Generally, the bridge deterioration process is affected by the explanatory variables, such as traffic volume, climatic condition, and material properties [1]. However, the most explanatory variables are unable to be captured by filed bridge inspection. Therefore, deterioration is often characterised by using probabilistic models to predict uncertain deterioration in condition state variation at a given discrete time and explanatory variables. The probabilistic models can be grouped into two categories: state-based and time-based model. The state-based models predict long-term bridge performance using transition probability obtained from the difference between the two condition states at a given discrete time interval. The most common example is Markov chain models. Time-based models, on the other hand, employ probability density function of time, referring to the state duration time required for a bridge element to deteriorate from an initial condition state to its next specified state. Parametric, semi-parametric and non-parametric models have been proposed to generate the probability distribution function of transition time [2]. 
The challenge for probabilistic modelling is how to generate a reliable transition probability. For state-based model, several methods have been developed to calculate the transition probability, such as the expected-value method [3], poisson and binomial regression [4], and ordered probit model [5]. However, these methods have a most critical common shortcoming is that they are only workable when given condition data has ideal distribution over the time [6]. For a time-based model, the most common critical shortcoming in generating transition probability are that they require frequent inspection of condition ratings over a long period and has obvious transition changes in given condition data. Moreover, an element-level inspection record usually suffers the problem of various situations of condition rating data distributions due to insufficient inspection records. Therefore, using a stand-alone model such as state- or time-based model is unable or unreliable to predict longterm bridge performance due to the shortcomings of these models outlined above.

In order to minimise the above-mentioned shortcomings, this paper presents an integrated method incorporating both state- and time-based model. This integrated method is effective as compared to the stand-alone model because a selection process is embedded to the integrated method to automatically select a suitable prediction approach (either state- or time-based) for a given situation of available condition data. Moreover, a previously developed Backward Prediction Model (BPM) is also ultilised to generate the missing historical data for insufficient condition data. To verify the performance of the integrated method, four different types of situations regarding the amount and distribution of available condition rating data are predefined. In this study, the available inspection records are the concrete element 59C-Pilecap in bridge substructure. This type of situations is selected as a benchmark example to demonstrate the advantage of the integrated method in predicting long-term performance using insufficient data. This example is that time-based model uses the BPM-generated data together with available inspection data to estimate the transition probability and thereby predicting long-term bridge element performance. The outcome of this example is also used to identify the advantages of incorporating BPM in the integrated method.

\section{Methodology}

The simplified integrated method is presented in Figure 1. The major component of this method includes categorisation, calculating Overall Condition Ratings (OCRs), selection process, and process of time-based model.

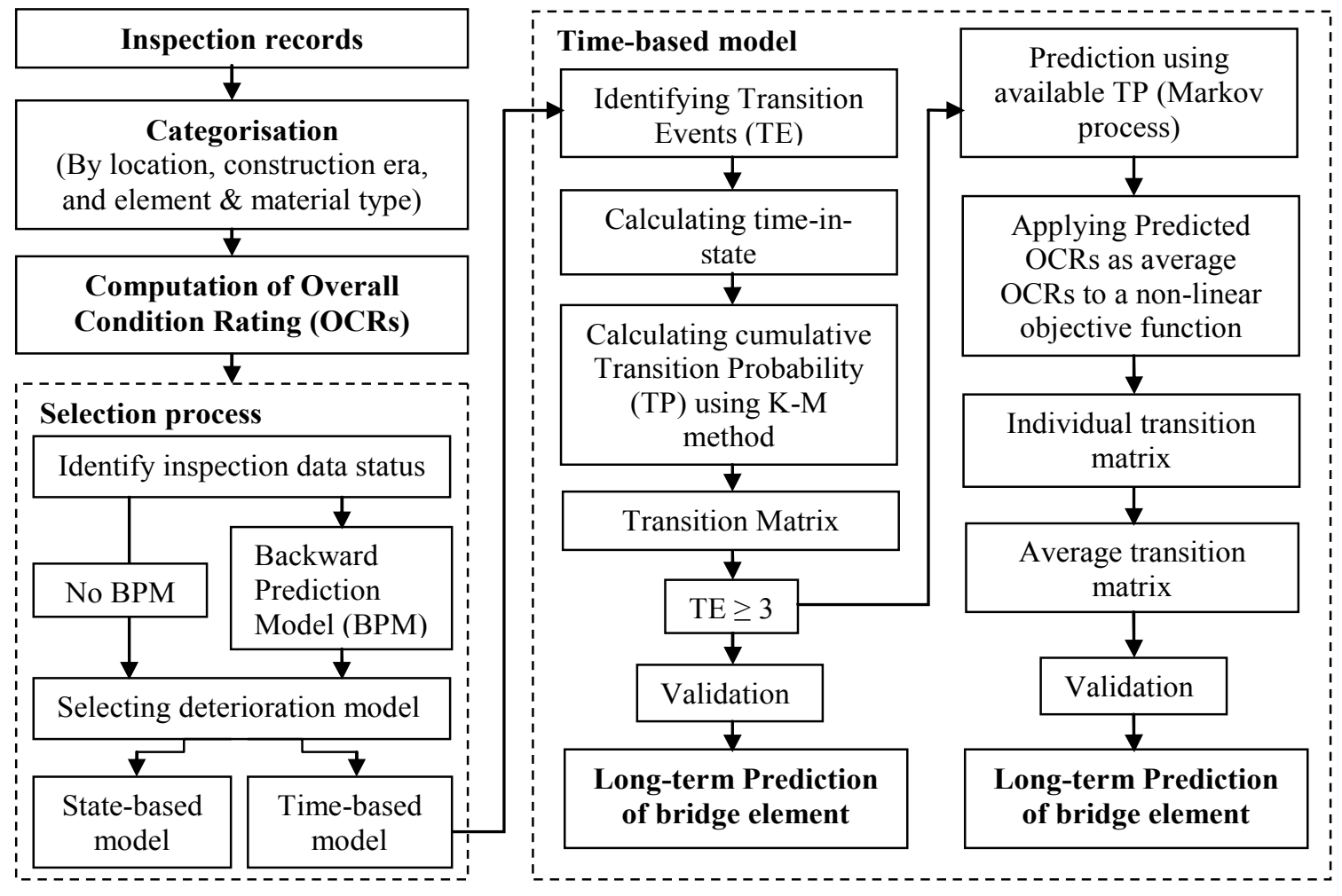

Fig. 1: The components and procedures of the proposed integrated method 
The inspection records obtained from Queensland department of Transport and Main Roads (QTMR), Australia. The categorisation of bridge inspection records is carried out according to the classification of structural deterioration obtained from the QTMR. On this research, the available inspection records only deals with classification in relation to bridge location, construction era, element type and material type. Note that construction era is also considered as one categorisation because the quality of bridge construction materials and construction methods in the last few decades has been continuously improved as compared to the earlier constructed structures. Once categorisation process completed, the OCRs is computed using the following Equation:

$$
O C R=\frac{q_{1} w_{1}+q_{2} w_{2}+q_{3} w_{3}+q_{4} w_{4}}{q_{1}+q_{2}+q_{3}+q_{4}}
$$

where, $q_{1}, q_{2}, q_{3}$ and $q_{4}$ are element quantities in condition states (CSs) 1, 2, 3 and 4, respectively, and $w_{1}, w_{2}, w_{3}$ and $w_{4}$ are weighting factors for each condition state [7]. Four CSs are in the order of 1 to 4 (excellent to very poor), and in this study they represent the bridge condition ratings $100 \%$, $70 \%, 50 \%$ and $20 \%$ in a descending order.

The selection process is developed to identify the status of available inspection records, and in turn select a most suitable prediction approach based on four different alternatives. Time-based model as the first alternative is applied when available inspection records satisfied the input requirement of a time-based model. State-based model as the second alternative is used when available inspection records are only workable using state-based model. The third and last alternatives are applied when available inspection records are insufficient and unable to be used by either time-based or statebased model. With insufficient inspection records, the BPM is employed to generate missing historical condition ratings. The BPM-generated condition ratings together with available inspection records are only workable using time-based model, is consider as third alternative. The last alternative is only workable using state-based model when BPM-generated condition ratings together with available inspection records [8]. In this paper, the third alternative, time-based model using BPM-generated condition rating together with available inspection records is selected as the benchmark example to demonstrate the advantage of the proposed integrated method.

The mechanism of the BPM is depicted in Figure 2. The BPM is based on the Artificial Neural Networks (ANN) to establish a correlation between available condition rating records (year $t_{p}-t_{p n}$ ) and corresponding years' non-bridge factors. The non-bridge factors, such as climate and

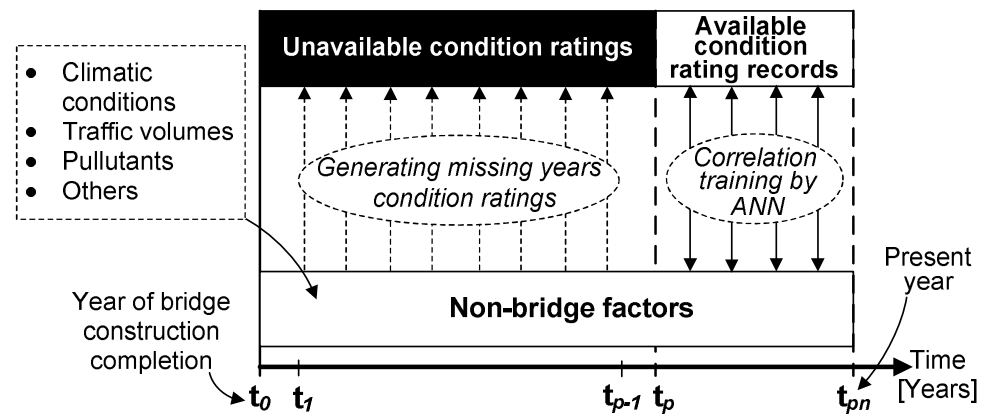

Fig.2: Mechanism of the BPM [9] environmental condition changes and traffic volume increases, are obtained from relevant information authorities. These non-bridge factors directly and indirectly affect the variation of the bridge conditions and thus the deterioration rate. Once the correlation is established by neural networks, the missing historical condition ratings (year $t_{1}-t_{p-1}$ ) can be generated by using the non-bridge factors [9].

Once the BPM-generated missing

historical condition data together with available inspection records are ready, the time-based model is able to generate transition probability. The process of the time-based model as illustrated in Figure 1 is detailed herein. The time-based models require sequential changes (past $i$, current $j$ and future $k$ ) in condition ratings to define state transition events and the corresponding transition times. The value of sequential condition rating $j$ is transferred to a lower condition state $k=j-1$. This is defined as a state transition event. Each transition event can be denoted as TE $(j, k)[10]$. The transition time is defined as the time spend for an element in an original condition state deteriorating to the next lower condition state in the condition rating scale. In this study, the QTMR inspection records are only available transition event TE $(1,2)$ and actual time of this transition event is unknown because visual inspection activities are performed periodically over an observation period, and the related time data are considered "multiply censored" [11]. Censored data means that the observed event does not changing in condition states during the observation 
period. A censored event can become a transition event after a specific time (right censored, or Rcensored), before a specific time (left censored, or L-censored), or both (interval censored, Icensored). In normal practice, the transition event is assumed to occur at the middle of the observation period if it is observed between two consecutive condition states [11]. The time-in-state includes the time spent for censored events and transition events. Table 1 is only used for computing the time-in-state for TE $(1,2)$ when the construction or MR\&R work is performed at known time.

Table 1: Time-in-state for TE $(1,2)$ from CS1 to CS2

\begin{tabular}{|c|c|c|c|c|}
\hline \multicolumn{3}{|c|}{ Condition state } & Time-in-state & Data type \\
\hline$i$ & $j$ & $k$ & $\mathrm{~T}_{\mathrm{j}}$ & \\
\hline Construction yr & $\mathrm{CS} 1$ & $\mathrm{CS} 2$ & $\mathrm{~T}_{\mathrm{ij}}+\mathrm{T}_{\mathrm{jk}} / 2$ & Uncensored \\
\hline Construction yr & CS1 & CS1 & $\mathrm{T}_{\mathrm{ij}}+\mathrm{T}_{\mathrm{jk}}$ & R-censored \\
\hline
\end{tabular}

The time-based models estimate the transition probability from the cumulative probability of the transition event within the transition time. In this study, the Kaplan and Meier methods is employed to estimate nonparametric reliability function with
conding transition times and events respect to the cumulative transition probabilities and the corresponding transition times and events
$[10]$. According to DeStefnano and Grivs (1998), the equations for calculating the reliability of a bridge element and estimating the cumulative transition probability are presented below:

$$
\begin{aligned}
& \hat{R}\left(t_{x}\right)=\left[\left(r_{x}-1\right) / r_{x}\right] \times R_{x-1} \\
& \operatorname{TP}\left(t_{x}\right)=1-\hat{R}\left(t_{x}\right)
\end{aligned}
$$

where $\hat{R}\left(t_{x}\right)=$ estimated reliability of a bridge element at time $t_{x}$ (years); $r_{x}=$ reversed rank order of all time value observed within the sample interval; and $\operatorname{TP}\left(t_{x}\right)=$ cumulative transition probability for all $x=1,2,3, \ldots y$ th sample observation in ascending time order; and $R_{0}=1$ at $t=0$.

As shown in Figure 1 under "time-based model", more than 3 state transition events, i.e. TE $\geq 3$ (in this study, TE $(1,2)$, TE $(2,3)$ and TE $(3,4))$ is required to compute the transition probabilities. However, when the state transition events are less than 3 , i.e. only TE $(1,2)$ or TE $(1,2)$ and TE $(2$, 3 ), the Markov process needs to be employed to generate an individual transition probability matrix. In this process, the condition ratings generated from the time-based model are assumed as the average overall condition ratings $A(t)$. The transition probability is obtained by minimising the difference between average condition rating $A(t)$ and estimated condition rating $E(t)$. A non-linear programming objective function is described as follow:

$$
\operatorname{Min} \sum_{t=1}^{N}|A(t)-E(t)| \text { subject to } 0 \leq P(i) \leq 1, i=1,2,3 \ldots ., U \text {. }
$$

where, $N=$ the number of years in one age group; and $U=$ the number of unknown probabilities; $E(t)=$ the estimated OCRs at age $t$ by the Markov chain method [8]

The accuracy of the transition probability depends on the closeness of the average OCRs and the OCRs predicted by Markov chain method. The Chi-square goodness of fit test is used to validate the accuracy of the transition probability. The calculation formula for the Chi-square method is given below:

$$
\chi^{2}=\sum_{i=1}^{k} \frac{\left(E_{i}-A_{i}\right)^{2}}{E_{i}}
$$

where, $\chi^{2}=$ a Chi-square distribution with $k$-1 degrees of freedom, $E_{i}=$ value of the condition rating in year $i$ predicted by the Markov chain method, $A_{i}=$ value of condition rating in year $i$ obtained from time-based model, and $k=$ number of prediction years. 


\section{Benchmark Example}

The benchmark example concrete element type 59C-Pilecap/Footing in bridge substructures has

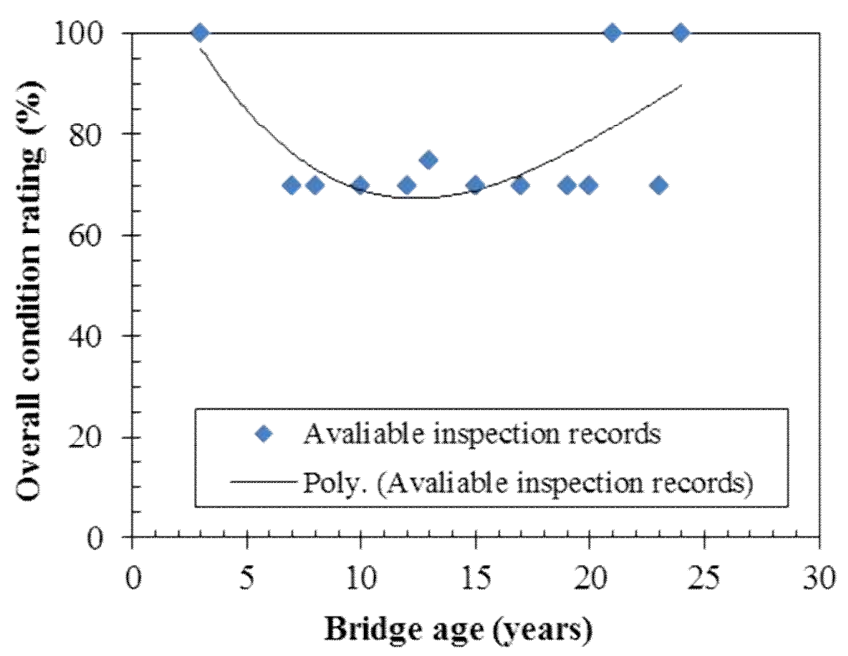

Fig. 3: Available inspection records total of 7 bridges and 14 inspection records. This is a foundation element and used to support pile bases at piers and abutments. Therefore, it is important to select this element as an example to predict long-term performance of bridge element. Figure 3 shows the distribution of available inspection data and $3^{\text {rd }}$-order polynomial regression curve. As shown in the figure, the regression curve presents an unrealistic average condition rating deterioration rate and the available inspection records have only one transition event TE (1, 2 ). In order to obtain a workable transition probability for concrete element type 59C, the BPM is employed to generate the missing historical condition ratings for bridges ID\#XX142XX, \#XX144XX and \#XX147XX. Consequently, the BPM generated missing condition ratings together with the available data provide a total of 4 transition events TE (1, 2) to be used in the time-based model to generate transition probability.

The Kaplan and Meier method is used to generate the non-parametric values with regard to the cumulative transition probabilities corresponding to transition times and specific transition events. The example of estimating the cumulative transition probabilities of TE $(1,2)$ for concrete element

Table 2: Calculating cumulative Transition Probability

\begin{tabular}{cccccc}
\hline $\begin{array}{c}\text { Observation } \\
(x)\end{array}$ & $\begin{array}{c}\text { Time-in- } \\
\text { state }\left(t_{x}\right)\end{array}$ & Model & $\begin{array}{c}\text { Rank } \\
\left(r_{x}\right)\end{array}$ & Reliability & $\begin{array}{c}\text { Cumulative } \\
\text { TP }\end{array}$ \\
\hline 1 & 2.50 & 1 & 5 & 0.800 & 0.200 \\
2 & 5.96 & 1 & 4 & 0.600 & 0.400 \\
3 & 10.50 & 1 & 3 & 0.400 & 0.600 \\
4 & 14.50 & 1 & 2 & 0.200 & 0.800 \\
5 & 24.75 & 0 & 1 & 0.200 & 0.800 \\
\hline
\end{tabular}
type 59C is illustrated in Table 2. In this table, Model 0 is defined as a censored event, and Model 1 is defined as a transition event. Reliability is calculated by Equation (2) and transition probability is calculated by Equation (3). A linear regression is used to calculate the uniform distribution function for concrete element type 59C as presented in

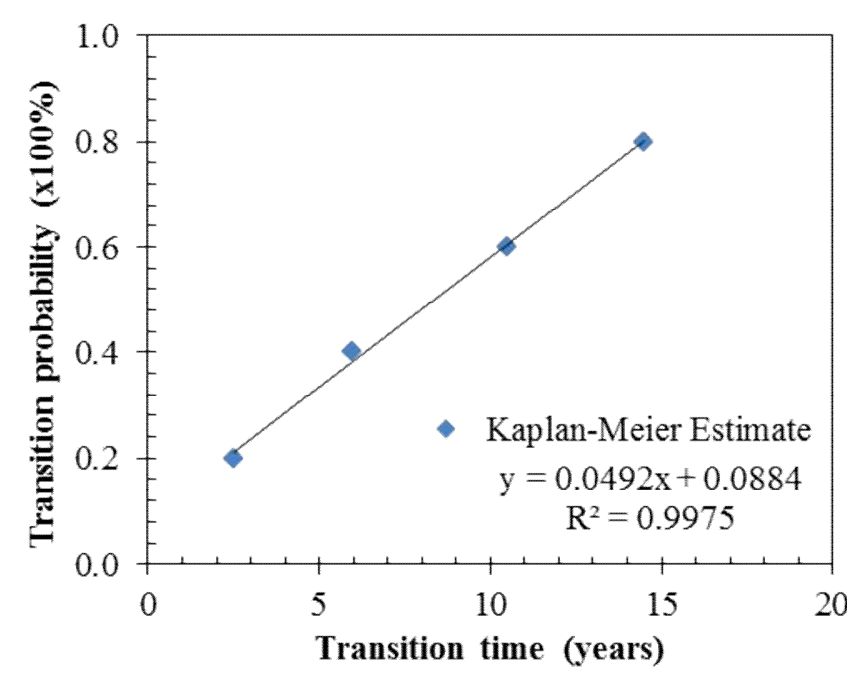

Fig. 4: Element type 59C deterioration model
Figure 4. The linear regression parameters are then used to generate the transition probability for transition event TE $(1,2)$ for this concrete element type.

As an example, the transition probability for TE $(1,2)$ for bridge substructure element 59C-Bridge\#XX48XX is shown in Figure 5. Note that these generated transition probabilities can only be used to predict condition ratings from $\mathrm{CS} 1$ to $\mathrm{CS} 2$. For future and long-term prediction, the Markov chain method has to be used in this case to generate an individual transition probability for each element. Table 3 summarises the predicted OCRs using transition probabilities obtained from the time-based model for bridge element 59C-Bridge\#XX48XX. The predicted OCRs are considered as the 
average condition rating $A(t)$ to be used in Equation (4) to generate the individual transition probability.

$$
\begin{array}{c|cccc|}
\multicolumn{1}{c}{\mathbf{1}} & \mathbf{2} & \mathbf{3} & \mathbf{4} \\
\mathbf{1} & 0.764 & 0.471 & 0 & 0 \\
\mathbf{2} & 0 & 1.000 & 0 & 0 \\
\mathbf{3} & 0 & 0 & 1.000 & 0 \\
\mathbf{4} & 0 & 0 & 0 & 1.000
\end{array}
$$

Transition probability for bridge age 4

\begin{tabular}{l|cccc|} 
& $\mathbf{1}$ & $\mathbf{2}$ & $\mathbf{3}$ & $\mathbf{4}$ \\
$\mathbf{1}$ & 0.666 & 0.334 & 0 & 0 \\
$\mathbf{2}$ & 0 & 1.000 & 0 & 0 \\
$\mathbf{3}$ & 0 & 0 & 1.000 & 0 \\
$\mathbf{4}$ & 0 & 0 & 0 & 1.000
\end{tabular}

Transition probability for bridge age 6

\begin{tabular}{l|cccc|} 
& $\mathbf{1}$ & $\mathbf{2}$ & $\mathbf{3}$ & $\mathbf{4}$ \\
$\mathbf{1}$ & 0.715 & 0.285 & 0 & 0 \\
$\mathbf{2}$ & 0 & 1.000 & 0 & 0 \\
$\mathbf{3}$ & 0 & 0 & 1.000 & 0 \\
$\mathbf{4}$ & 0 & 0 & 0 & 1.000
\end{tabular}

Transition probability for bridge age 5

\begin{tabular}{l|cccc|}
\multicolumn{1}{c}{} & $\mathbf{1}$ & $\mathbf{2}$ & $\mathbf{3}$ & $\mathbf{4}$ \\
$\mathbf{1}$ & 0.616 & 0.384 & 0 & 0 \\
$\mathbf{2}$ & 0 & 1.000 & 0 & 0 \\
$\mathbf{3}$ & 0 & 0 & 1.000 & 0 \\
$\mathbf{4}$ & 0 & 0 & 0 & 1.000
\end{tabular}

Transition probability for bridge age 7

$$
\begin{array}{l|cccc} 
& \mathbf{1} & \mathbf{2} & \mathbf{3} & \mathbf{4} \\
\mathbf{1} & 0.567 & 0.433 & 0 & 0 \\
\mathbf{2} & 0 & 1.000 & 0 & 0 \\
\mathbf{3} & 0 & 0 & 1.000 & 0 \\
\mathbf{4} & 0 & 0 & 0 & 1.000
\end{array}
$$

Transition probability for bridge age 8

Fig. 5: Transition probabilities for TE $(1,2)$ obtained from the time-based model for bridge element 59C-Bridge\#XX48XX

Table 3: Predicted OCRs using transition probability obtained from the time-based model

\begin{tabular}{ccccccc}
\hline & \multirow{2}{*}{ Initial input } & \multicolumn{5}{c}{ Predicted values using transition probability obtained from } \\
the time-based model \\
\hline Year & 1999 & 2000 & 2001 & 2002 & 2003 & 2004 \\
Bridge age & 3 & 4 & 5 & 6 & 7 & 8 \\
OCRs (\%) & 100.00 & 92.92 & 86.38 & 80.90 & 76.72 & 73.81 \\
\hline
\end{tabular}

\section{Results and discussion}

The purpose of this study is to demonstrate the capability of applying the BPM in the integrated method by a benchmark example. Detail result of this example is presented in this section. Figure 6 presents the individual transition probability matrix for bridge

\begin{tabular}{|c|c|c|c|c|}
\hline & 1 & 2 & 3 & 4 \\
\hline 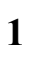 & 0.795 & 0.205 & 0 & 0 \\
\hline 2 & 0 & 0.546 & 0.454 & 0 \\
\hline 3 & 0 & 0 & 1.000 & 0 \\
\hline . & 0 & 0 & 0 & 1.000 \\
\hline
\end{tabular}
element 59C-Bridge\#XX48XX. The

Fig. 6: Individual transition matrix

transition probability matrix is obtained by using the time-based model incorporating the Markov chain method.

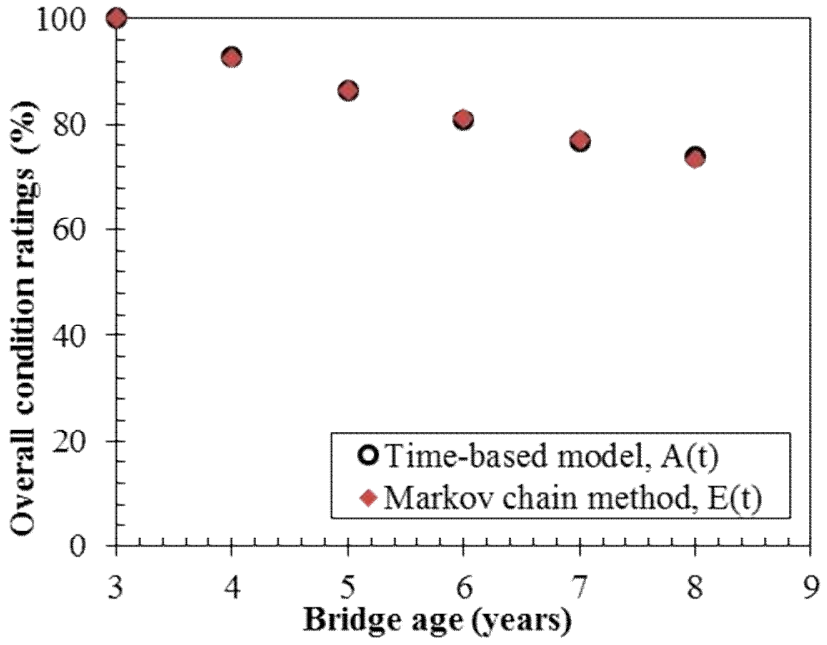

Fig. 7: Comparison between the average $A(t)$ and predicted $E(t)$ 
Figure 7 compares the average condition rating $A(t)$ obtained from the time-based model and the predicted condition ratings $E(t)$ from the Markov chain method for bridge element $59 \mathrm{C}$ -

Bridge\#XX48XX. As the shown in figure, the predicted OCRs from the Markov chain method are very close to the average condition ratings. Moreover, Table 4 summaries the degrees of freedom, critical $x^{2}$ values at significance level $\alpha=0.05$ and that obtained from perdition method. The comparison shows that the estimated $x^{2}=0.0001$ is much smaller than that at significance level $\alpha=$ 0.05 . This means that generated transition probability are well acceptable to predict long-term bridge performance.

Table 4: Comparison of the $x^{2}$ values at significance level $\alpha=0.05$

\begin{tabular}{ccccc}
\hline Element type & Construction era & Degrees of freedom & $x^{2}$ critical $(\alpha=0.05)$ & $x^{2}$ value \\
\hline $59 \mathrm{C}$ & $1981-2000$ & 5 & 11.07 & 0.0001 \\
\hline
\end{tabular}

Once the transition probability is confirmed, the long-term bridge element prediction can be simply conducted. Figure 8 shows long-term prediction for Bridge\#XX48XX. It is based on the latest inspection record as an initial condition state vector from this bridge, by which the element condition rating for future 25 years are predicted without MR\&R. The long-term prediction outcome presented in the figure shows both element quantity and OCR each year.

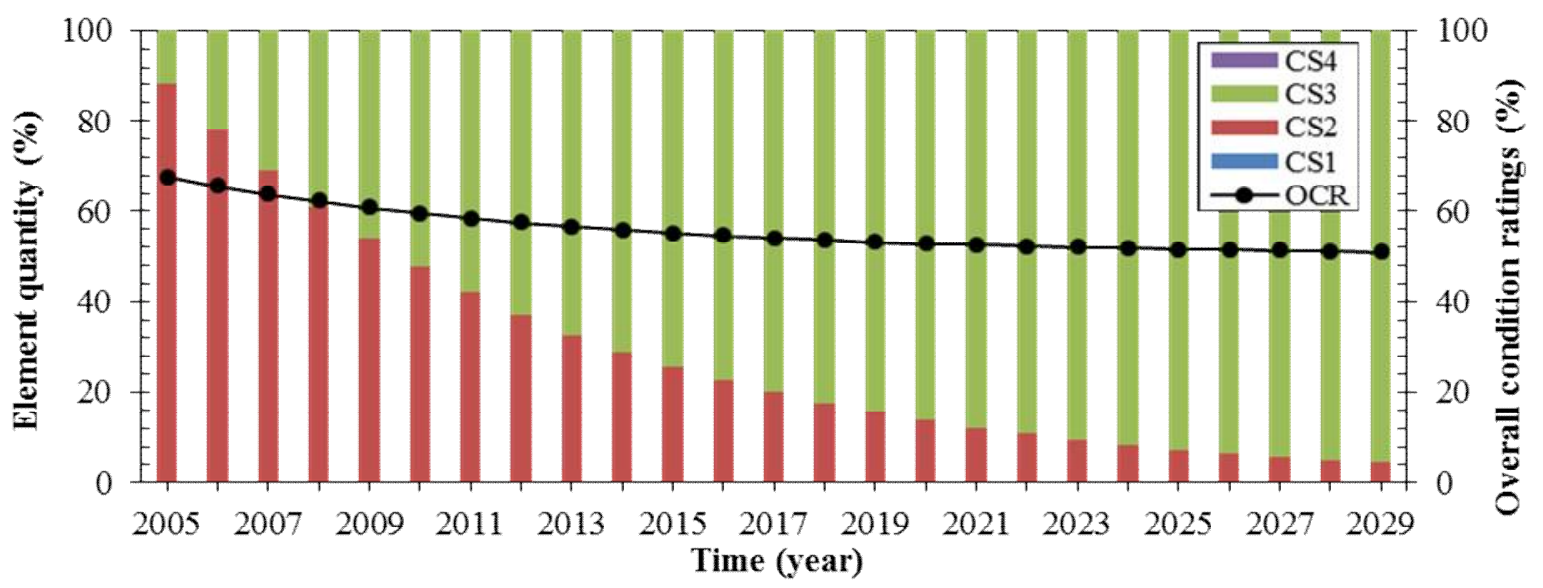

Fig. 8: Long-term prediction by element quantities and OCRs (59C-Bridge\#XX48XX)

This study demonstrates the advantage of integrated method and capability of the BPM in predicting long-term performance of bridge element. The advantages can be summarised as follows: (1) the integrated method categorises the bridge elements according to bridge location, construction era, element type and material type, by which similar elements are grouped together to identify common deterioration patterns; (2) The benchmark example demonstrates that the BPM-generated historical condition ratings together with available inspection records as input can be used by the time-based model; (3) The BPM-generated data together with available inspection records can improve the reliability of estimating the transition probabilities and thereby improving the reliability of long-term bridge element prediction.

\section{Conclusion}

This study presents an integrated method using typical probabilistic deterioration modelling techniques to provide alternative workable solution for various situations of available condition data. The most prominent techniques, such as state-based and time-based models are employed in this integrated method to predict long-term bridge element performance. Moreover, the selection process is employed to automatically select a suitable prediction approach for a given situation of condition data. Application of the selection process in the integrated method leads more effective prediction outcomes as compared to stand-alone model (i.e. state-based or time-based model). The BPM is also used in the proposed integrated method to generate the missing historical data for insufficient data scenarios. One situation is selected as a benchmark example to demonstrate the workability of the time-based model in conjunction with BPM in the integrated method. The outcome shows that the integrated method is effective with insufficient historical condition ratings 
are only available. On the basis of this study, further investigation in necessary with more case studies in order to demonstrate the reliability and accuracy of the proposed integrated method.

\section{Acknowledgements}

The authors acknowledge the financial support provided by the Australian Research Council through an ARC Linkage Project (LP0883807). The authors also wish to thank the industry partners: Queensland Department of Transport and Main Roads and Gold Coast City Council for their financial and in-kind support.

\section{Reference}

[1] MAUCH M., and MADANAT S., "Semiparametric Hazard Rate Models of Reinforced Concrete Bridge Deck Deterioration", ASCE Journal of Infrastructure Systems, Vol. 7, No. 2, 2001, pp. 49-57.

[2] MORCOUS G., and AKHNOUKH A., "Stochastic Modelling of Infrastructure Deterioration: An Application to Concrete Bridge Decks", International Conference on Computing and Decision Making in Civil and Building Engineering, Montreal, Canada, June14-16, 2006.

[3] JIANG Y., SAITO M., and SINHA K.C., "Bridge performance prediction model using the Markov chain", Transportation Research Record, 1988, pp.25-32.

[4] MADANAT S., and IBRAHIM W.H.W., "Poisson Regression Models of Infrastructure Transition Probabilities", Journal of Transportation Engineering, Vol. 121, No. 3, 1995, pp.267-272.

[5] MADANAT S., MISHALANI R., and IBRAHIM W.H.W., "Estimation of Infrastructure Transition Probabilities from Condition Rating Data", Journal of Infrastructure Systems, Vol. 1, No. 2, 1995, pp.120-125.

[6] MISHALANI R.G., and MADANAT S.M., "Computation of Infrastructure Transition Probabilities Using Stochastic Duration Models", Journal of Infrastructure Systems, Vol. 8, No. 4, 2002, pp.139-148.

[7] THOMPSON P., and SHEPARD R., "AASHTO Commonly-Recognized Bridge Elements", Workshop on National Commonly Recognized Measures for Maintenance, June, 2000.

[8] BU G.P., LEE J.H., GUAN H., BLUMENSTEIN M., and LOO Y.C., "Development of an Integrated Method for Probabilistic Bridge Deterioration Modelling", ASCE Journal of Performance of Constructed Facilities, 2012, Submitted.

[9] LEE J.H., SANMUGARASA K., LOO Y.C., and BLUMENSTEIN M., "Improving the Reliability of a Bridge Management System (BMS) using an ANN-based Backward Prediction Model (BPM)", Journal of Automation in Construction, Vol. 17, No. 6, 2008, pp.758-772.

[10] DESTEFANO P. D., and GRIVAS D. A., "Method for estimating transition probability in bridge deterioration models", Journal of Infrastructure System, Vol. 4, No. 2, 1998, pp.56-62.

[11] MORCOUS G., LOUNIS Z., and CHO Yong., "An Integrated System for Bridge Management Using Probabilistic and Mechanistic Deterioration Models: Application to Bridge Decks." KSCE Journal of Civil Engineering, Vol. 14, No. 4, 2010, pp.527-537. 